\title{
Émile Guimet et la morsure du canard égyptien. Un curieux au musée de Boulaq
}

Émile Guimet and the Egyptology bug: a curious visitor at the Musée de Boulaq

\section{Thomas Lebée}

\section{OpenEdition}

\section{Journals}

Édition électronique

URL : http://journals.openedition.org/cel/409

DOI : $10.4000 /$ cel.409

ISSN : 2262-208X

Éditeur

École du Louvre

Édition imprimée

Date de publication : 1 octobre 2014

Référence électronique

Thomas Lebée, «Émile Guimet et la morsure du canard égyptien. Un curieux au musée de Boulaq », Les Cahiers de l'École du Louvre [En ligne], 5 | 2014, mis en ligne le 01 octobre 2014, consulté le 01 mai 2019. URL : http://journals.openedition.org/cel/409 ; DOI : 10.4000/cel.409

\section{(c) $(9) \odot$}

Les Cahiers de l'École du Louvre sont mis à disposition selon les termes de la licence Creative Commons Attribution - Pas d'Utilisation Commerciale - Pas de Modification 4.0 International. 


\title{
Cahiers de l'École du Louvre
} recherches en histoire de l'art, histoire des civilisations archéologie, anthropologie et muséologie

\section{Numéro 5. Octobre 2014}

\author{
Émile Guimet et la morsure du canard égyptien. \\ Un curieux au musée de Boulaq \\ Thomas Lebée
}

Article disponible en ligne à l'adresse :

http://www.ecoledulouvre.fr/cahiers-de-I-ecole-du-louvre/numero5octobre2014/Lebee.pdf

Pour citer cet article :

Thomas Lebée, « Émile Guimet et la morsure du canard égyptien. Un curieux au musée de Boulaq », Cahiers de l'École du Louvre. Recherches en histoire de I'art, histoire des civilisations, archéologie, anthropologie et muséologie [en ligne] $n^{\circ} 5$, octobre 2014 , p. 58 à 66.

\section{(c) creative \\ (‥) $\ominus$}

(C) École du Louvre

Cet article est mis à disposition selon les termes de la Licence Creative Commons Attribution - Pas d'utilisation commerciale - Pas de modification 3.0 non transposé. 


\section{Cahiers de l'École du Louvre recherches en histoire de l'art, histoire des civilisations archéologie, anthropologie et muséologie \\ Numéro 5. octobre 2014}

\section{Sommaire}

\section{Introduction}

L'archéologie en construction : objets, images, dispositions

Nathan Schlanger

La collection de vases grecs du marquis de Northampton (1790-1851).

Entre archéologie et sciences de la nature

Marie-Amélie Bernard.

L'artisanat touristique du Sud-Ouest des États-Unis. L'exemple des objets collectés par Alphonse Pinart à Santa Fe, à la fin du XIX ${ }^{\mathrm{e}}$ siècle Éloïse Galliard

Regard sur les statuettes hindoues et bouddhiques en bronze d'Indonésie. Leur rôle pour la connaissance de la civilisation javanaise ancienne et ses liens avec l'Asie du Sud et du Sud-Est

Mathilde Mechling 25-33

Le Tigre, le Louvre et l'échange de connaissances archéologiques visuelles entre la France et la Grande-Bretagne aux alentours de 1850

Mirjam Brusius p. $34-46$

Le regard des photographes commerciaux. Quelques clichés du fonds égyptien de la Collection Fouad Debbas à l'étude.

Yasmine Chemali - Anne-Hélène Perrot

Émile Guimet et la morsure du canard égyptien.

Un curieux au musée de Boulaq

Thomas Lebée

L'exposition préhistorique de la Galerie de l'Histoire du travail en 1867. Organisation, réception et impacts 


\section{Émile Guimet et la morsure du canard égyptien. Un curieux au musée de Boulaq}

\section{Thomas Lebée}

"Le canard égyptien [...] est un animal dangereux. Il vous accueille bénignement, mais si vous vous laissez prendre à son air innocent et que vous le pratiquez familièrement, vous êtes perdu : un coup de bec, il vous inocule son venin, et vous voilà égyptologue pour la vie ${ }^{1}$. »

Auguste Mariette

\section{«Un voyage de touriste en Égypte »}

Quand il partit en Égypte, en 1865, Émile Guimet n'était pas encore l'orientaliste distingué qu'il devint par la suite. Cet industriel et philanthrope lyonnais avait alors vingt-neuf ans; il participait à l'entreprise familiale, qui produisait du bleu outremer. Émile Guimet publia le récit de son voyage quelques années plus tard; il écrivit lui-même qu'il effectua "comme tout le monde, un voyage de touriste en Égypte ${ }^{2}$ ». C'est en effet à cette époque que naît le tourisme organisé autour de la Méditerranée et plus particulièrement en Égypte, le tourisme "domestique » et européen s'étant déjà fortement développé au début $\mathrm{du} \mathrm{XIX}^{\mathrm{e}}$ siècle. Des compagnies comme celle de Thomas Cook organisaient des croisières régulières sur le Nil pour la bourgeoisie aisée d'Europe, alors que se développaient les guides de voyage consacrés à l'Égypte, notamment les fameux guides Murray, Baedeker et Joanne \& Isambert ${ }^{3}$. Si l'inauguration du canal de Suez en 1869 et le développement des liaisons qui s'ensuivit facilitèrent encore l'organisation de ces voyages, un certain nombre d'Européens avaient choisi l'Égypte comme destination d'un voyage d'agrément dès le milieu du siècle. Le " comme tout le monde " d'Émile Guimet est révélateur ; on pensera par exemple aux récits de Gustave Flaubert ${ }^{4}$ et de son compagnon de voyage Maxime Du Camp 5 , ou à celui d'Arthur Rhoné6. Le récit d'Émile Guimet mentionne, presque à chaque étape, la présence de guides polyglottes qui se consacrent au service des touristes moyennant pourboire et s'adaptent à leur langue (français, italien, allemand, grec); c'est la preuve d'un tourisme régulier et international.

Cet article reprend certains éléments de notre mémoire : Le musée d'antiquités égyptiennnes de Būlāq. Faire connaître et aimer l'Égypte ancienne au XIXe siècle, mémoire d'étude de l'École du Louvre soutenu en juin 2013 sous la direction d'Élisabeth David et d'Elsa Rickal, que nous tenons à remercier pour leurs encouragements constants.

1. Gaston Maspero, «Mariette (1821-1881). Notice biographique », Auguste Mariette, Fuvres diverses (Bibliothèque égyptologique 18), t. 1, Paris, Ernest Leroux, 1904, pp. I-CCXXIV (p. VI ; nous remercions Élisabeth David d'avoir retrouvé I'origine de cette anecdote). Une citation très similaire est d'ailleurs prêtée à Emmanuel de Rougé : Gaston Maspero, « Notice biographique du vicomte Emmanuel de Rougé », dans Emmanuel de Rougé, Fuvres diverses (Bibliothèque égyptologique 21), t. 1, Paris, Ernest Leroux, 1907, pp. I-CLVI (p. VI ; nous devons cette précision à Kimberley Harthoorn).

2. Émile Guimet, Le jubilé du musée Guimet. Vingt-cinquième anniversaire de sa fondation. 1879-1904, Paris, Ernest Leroux, 1904, p. 10.

3. Pour les $1^{\text {res }}$ éditions : John Gardner Wilkinson, Handbook for Travellers in Egypt, Londres, John Murray, 1847; Adolphe Joanne \& Émile Isambert, Itinéraire descriptif, historique et archéologique de l'Orient, Paris, Hachette et Cie, 1861 ; Karl Baedeker, Egypt. Handbook for travellers, t. 1 Lower Egypt, with the Fayûm and the Peninsula of Sinai, Leipzig, Karl Baedeker Londres, Dulau \& $C^{\circ} ., 1878$.

4. Gustave Flaubert, Voyage en Orient, Paris, Gallimard, 2006.

5. Maxime Du Camp, Égypte, Nubie, Palestine et Syrie. Dessins photographiques recueillis pendant les années 1849, 1850 et 1851, Paris, Gide et J. Baudry, 1852.

6. Arthur Rhoné, L'Égypte à petites journées. Études et souvenirs. Le Kaire et ses environs, Paris, Ernest Leroux, 1877 (récit d'un voyage en 1864). 
Grâce au récit d'Émile Guimet ${ }^{7}$, nous connaissons les conditions précises de son voyage, ce qui nous laisse un témoignage remarquable de ses visites au musée de Boulaq. Revenons brièvement sur l'organisation du séjour. Émile Guimet partit de Marseille le 9 novembre 1864; il arriva à Alexandrie le 17 et visita de grandes villes de la côte et du delta du Nil : Zagazig, Ismailia et Port-Saïd. Il resta ensuite au Caire du 26 novembre au 10 décembre, et en profita pour visiter la ville et sa région. C'est au cours de ce séjour qu'il effectua ses deux premières visites à Boulaq, le 28 novembre et le 9 décembre, et réalisa deux excursions à Suez, du 30 novembre au 2 décembre et à Alexandrie du 5 au 7 décembre. Il prit ensuite une place sur un des bateaux qui remontaient le Nil le 10 décembre pour visiter la Haute-Égypte jusqu’à Philæ. Revenu au Caire le 29 décembre, il y passa quelques jours consacrés à diverses visites dont une tentative le 5 janvier 1865 pour revenir au musée de Boulaq, fermé le vendredi ; il y revint avec succès le 6 janvier - pour quitter la ville le 8 janvier, embarquer à Alexandrie le 9 janvier et toucher terre à Marseille le 16 janvier.

Chaque escapade demandait une certaine préparation : outre les provisions, la présence d'un drogman, à la fois guide, interprète, organisateur, de porteurs, d'attelage, etc. pouvait être une nécessité ou un simple agrément. Notons également que les visites concernaient tous les aspects de l'Égypte : Émile Guimet manifestait autant d'intérêt pour les sites antiques que pour le pittoresque des mœurs contemporaines, ou même pour la controverse sur la viabilité du canal de Suez encore en cours de percement. Son voyage lui permit de découvrir le patrimoine archéologique égyptien, qui était alors sous la responsabilité d'un seul homme : Auguste Mariette, directeur du Service de conservation des antiquités de l'Égypte.

\section{L'ambitieux projet d'Auguste Mariette}

Le Service de conservation des antiquités de l'Égypte fut créé à l'initiative d'Auguste Mariette ${ }^{8}$, égyptologue français qui se mit au service des vice-rois d'Égypte pour fonder une institution publique capable de protéger et mettre en valeur les richesses archéologiques égyptiennes. Jusqu’en 1858, date de la création du Service, les dispositions juridiques établies au début du siècle pour protéger le patrimoine égyptien ${ }^{9}$ étaient appliquées sans grande rigueur et les antiquités qui échappaient aux fouilles clandestines, et revenaient au souverain, étaient offertes avec libéralité aux diplomates étrangers.

Le Service, qui dépendait directement du vice-roi, reçut pour mission d'ouvrir des chantiers de fouille à travers tout le pays, notamment grâce à la corvée, et de créer un musée pour en abriter les fruits - le directeur du Service étant aussi directeur du musée. C'est donc avec des objectifs ambitieux qu'Auguste Mariette se mit à la tâche. "Seul maître du sol antique de l'Égypte, Mariette voulut l'exploiter sur un plan grandiose : il l'attaqua sur trente-sept points à la fois, de l'embouchure du Nil à la première cataracte ${ }^{10}$ ». Pourtant, il ne disposait que de ressources dérisoires. Les fonds alloués au Service, dépendant des caprices du vice-roi, lui permirent d'engager seulement une demi-douzaine de collaborateurs ${ }^{11}$. Avec cette équipe réduite, il dut diriger et publier ses fouilles, ce qui représentait déjà une masse de travail écrasante, mais aussi créer un musée ex nihilo (fig. 1).

7. É. Guimet, Croquis égyptiens. Journal d'un touriste, Paris, J. Hetzel, 1874.

8. Élisabeth David, Mariette Pacha. 1821-1881, Paris, Pygmalion, 1994.

9. Antoine Khater, Le régime juridique des fouilles en Égypte (RAPH 12), Le Caire, Institut français d'archéologie orientale, 1960, pp. 37-71.

10. G. Maspero, Guide du visiteur au musée de Boulaq, Boulaq, Musée de Boulaq, 1883, p. 18.

11. A. Mariette, Notice des monuments exposés dans les galeries provisoires du musée

d'antiquités égyptiennes de S.A. le vice-roi à Boulaq, Alexandrie, Mourès, Rey et Cie, 1864, p. 3. 


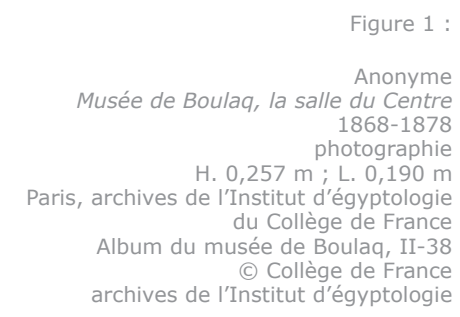

Encore fallait-il pour cela disposer d'un terrain. Le vice-roi promit à plusieurs reprises des monuments prestigieux pour accueillir le futur musée égyptien, qui devait prendre place parmi les institutions nouvelles du Caire rénové. Parmi les édifices proposés successivement, citons notamment le temple du Sphinx, à Giza ${ }^{12}$. Mais aucun de ces projets somptueux ne put être engagé. Auguste Mariette dut finalement se contenter d'une modeste parcelle au bord du Nil, dans le village de Boulaq, le port du Caire. Ce terrain ne lui fut attribué qu'en 1861, soit trois ans après la création officielle du musée.

Cette parcelle misérable offrait toutefois des avantages certains au Service: permettant de disposer d'un quai le long du Nil, elle facilitait l'acheminement des antiquités les plus encombrantes. De plus, si le musée était suffisamment proche du palais du vice-roi pour qu'Auguste Mariette pût aller y requérir quelque secours quand le besoin s'en faisait sentir, il en était assez éloigné pour rester à l'écart des préoccupations capricieuses du khédive ${ }^{13}$ et de sa générosité redoutable pour les collections nationales lors de visites diplomatiques étrangères. Enfin, sa situation dans les faubourgs du Caire, à un quart d'heure des hôtels du centre-ville $e^{14}$, en faisait un lieu facilement accessible aux touristes européens.

Toutefois, Boulaq était un faubourg qui comptait nombre d'entrepôts de céréales et d'alcool ${ }^{15}$. Les risques courus par le musée en cas d'incendie avaient

12. Patrizia Piacentini, «Archaeology and Archives: the Egyptian Museum in Egypt at the End of the Nineteenth Century », Francesco Raffaele et al., Recent Discoveries and latest Researches in Egyptology. Proceedings of the First Neapolitan Congress of Egyptology, Naples, June $18^{\text {th }}-20^{\text {th }}$ 2008, Wiesbaden, Harrassowitz, 2010, pp. 221-236, p. 222.

13. Titre accordé par le sultan ottoman au pacha d'Égypte en 1867, accompagné d'une large autonomie.

14. Émile Isambert, Itinéraire descriptif, historique et archéologique de l'Orient, t. 2, Malte, Egypte, Nubie, Abyssinie, Sinaï, Paris, Hachette et Cie, 1881, p. 352.

15. Eugène Grébaut, « Le transfert du Musée de Boulaq à Guizeh », Bulletin de l'Institut égyptien (3e série) 1, 1890 (1891), pp. 44-51. 
alarmé certains journalistes ${ }^{16}$. Le quartier avait aussi la réputation d'être mal famé ${ }^{17}$. Le voisinage du Nil n’allait pas non plus sans inconvénients : les salles étaient souvent fréquentées par des serpents ou des scorpions ${ }^{18}$. Les caprices de la crue étaient plus redoutables encore. Le musée fut plusieurs fois endommagé par les inondations; après la crue catastrophique de 1878 , où les salles furent inondées, il fut même détruit pour être rebâti à l'identique et rehaussé ${ }^{19}$.

Le manque chronique de moyens fut un autre ennemi du Service, malgré ses responsabilités et son ambition; les finances de l'Égypte étaient dans un état calamiteux et l'archéologie n'était pas le premier souci des vice-rois. Nous avons déjà rappelé que le personnel qui lui était alloué était bien insuffisant. Très vite, les salles furent saturées et le bâtiment exigu ne put contenir tous les objets mis au jour par les fouilles dignes d'être exposés, ce qui conduisit le Service à ouvrir à la visite un des magasins de stockage ${ }^{20}$ pour présenter les objets les plus encombrants. En remerciement de son engagement considérable pour les festivités de l'inauguration du canal de Suez (1869) $)^{21}$, Auguste Mariette reçut les moyens de construire deux petites salles, qui ne sont attestées qu'à partir de $1874^{22}$. Même dans les opérations courantes du Service, rien n'était simple : alors qu'il fallait fréquemment déplacer d'imposantes antiquités, tout le matériel (meubles, clous, palans, etc.) devaient être quémandés aux différents ministères, et parfois rendus après utilisation ${ }^{23}$.

\section{Faire connaître et aimer l'Égypte ancienne}

Malgré ces obstacles, Auguste Mariette mit tout en œuvre pour que son musée pût éveiller la curiosité des visiteurs pour l'Antiquité égyptienne. Ses méthodes étaient remarquables : dans les salles mêmes, chaque objet était accompagné d'un cartel qui en indiquait la provenance et l'époque. Ce dispositif a été commenté avec enthousiasme par divers observateurs ${ }^{24}$, ce qui prouve son originalité ; à titre de comparaison, les antiquités égyptiennes exposées au British Museum n’étaient alors accompagnées que d'un numéro ${ }^{25}$.

De même, Auguste Mariette lança un certain nombre de publications pour assister les visiteurs au cours de leur visite. La Notice des monuments exposés dans les galeries provisoires du musée d'antiquités égyptiennes de S.A. le vice-roi

16. [Anonyme], « The Boulaq Museum », The Athenæum 3125, 1887, p. 357. Cet article anglais proposa tout simplement, dans l'intérêt des collections, de transférer le contenu du musée au British Museum.

17. Le musée eut à affronter au moins deux cambriolages, voir Bibliothèque nationale de France, ms. N.A.F. 20177, fos 272-275.

18. É. David, op. cit. note 8, p. 119.

19. Henri Wallon, «Notice sur la vie et les travaux de François-Auguste-Ferdinand MariettePacha, membre ordinaire de l'Académie des Inscriptions et Belles-Lettres », CRAIBL (4e série) 11 , 1883 (1884), p. 45-163, voir appendice 26, p. 153.

20. Le «magasin $n^{\circ} 5 »$, dont l'ouverture est attestée dès 1868 , dans la $1^{\text {re }}$ édition de la Notice (A. Mariette, op. cit. note 11, pp. 231-256) et la fermeture notée dans la $3^{e}$ édition (Idem, Notice des monuments exposés dans les galeries provisoires du musée d'antiquités égyptiennes de S.A. le vice-roi à Boulaq, 3 éd., Paris, A. Franck, 1869, pp. 5-6).

21. Il accompagna par exemple les hôtes étrangers, dont l'impératrice Eugénie, lors d'une croisière en Haute-Égypte. On lui doit également l'intrique d'Aïda, commandé pour l'occasion mais créé en 1871. L'opéra se déroule dans une Égypte antique de fantaisie, voir Jean Humbert, « Aïda. Entre l'égyptologie et l'égyptomanie », L'Avant-scène opéra 4, 1976, pp. 9-14.

22. Comparer A. Mariette, op. cit. note 11, pp. 5-6 (annonce de I'ouverture prochaine des salles) et Id., Notice des monuments exposés dans les galeries provisoires du musée d'antiquités égyptiennes de S.A. le khédive à Boulaq $5^{e}$ éd., A. Mourès, Le Caire, 1874, pp. 67-68 (première attestation des nouvelles salles ouvertes au public).

23. Anne Léo, Auguste Mariette Pacha. Étude sur les débuts de l'archéologie égyptienne, mémoire de diplôme d'étude supérieure d'histoire, Faculté de Paris, 1946, t. 1 Texte, p. 91, mémoire inédit conservé à la bibliothèque de l'Institut d'égyptologie du Collège de France.

24. É. Guimet, op. cit. note 7, p. 124 ; vingt ans plus tard, il semble que ces cartels demeuraient une particularité appréciée : "Apart from the richness and number of the articles it contains, one great superiority enjoyed by this museum over all others is that the place whence every object comes is accurately known", "Mis à part la richesse et le nombre des objets qu'ils contient, une grande supériorité dont dispose ce musée sur tous les autres est que le lieu dont chaque objet provient est exactement connu », traduction de l'auteur; John Murray, A Handbook for Travellers in Lower and Upper Egypt, 7e éd., Londres, John Murray, 1888, p. 193. Les brouillons de ces cartels, réalisés à I'occasion de la rénovation de 1880, se trouvent à la Bibliothèque nationale de France (par exemple ms. N.A.F. 20177, fos 89, 92, 96 et 97).

25. Samuel Birch, Synopsis of the Content of the British Museum. Department of Oriental Antiquities: First and Second Egyptian Rooms, Londres, The British Museum, 1874, Note (non paginé). 
à Boulaq connut six éditions ${ }^{26}$ jusqu’à la mort d'Auguste Mariette. Ce guide du musée donne des explications globales sur l'Égypte ancienne, puis un commentaire plus ou moins détaillé, salle par salle, objet par objet. Il abordait avec pédagogie les débats de l'égyptologie à cette époque, et présentait même avec une certaine neutralité des théories que désapprouvait Auguste Mariette ${ }^{27}$. Cette Notice semble également avoir eu peu d'équivalent dans les musées égyptologiques contemporains: les guides contemporains du Louvre ${ }^{28}$, du British Museum ${ }^{29}$ ou des musées de Berlin ${ }^{30}$ et de Turin $^{31}$ comportaient des notices très sommaires et ne précisaient pas la provenance ou la datation des objets présentés ${ }^{32}$. Des recueils de photographies ${ }^{33}$ du musée furent aussi édités. Les Égyptiens contemporains étaient également pris en compte avec une réelle sollicitude ; Auguste Mariette écrivit à leur intention un guide particulier ${ }^{34}$ qu'il fit traduire en arabe ${ }^{35}$. Cette attention portée aux populations indigènes nous semble assez remarquable, même s'il est difficile d'évaluer le succès qu'elle a pu rencontrer.

Il ne faut pas oublier les Expositions universelles, qui représentaient aussi pour l'Égypte un moyen de faire connaître son patrimoine archéologique et ses collections auprès du grand public. Auguste Mariette s'investit considérablement dans la réalisation des pavillons égyptiens, notamment aux Expositions de 1862, $1867^{36}, 1873,1878^{37}$, concevant parfois lui-même les pavillons et prêtant certaines des pièces les plus précieuses du musée - ce qui n’alla pas sans mésaventures ${ }^{38}$ ou des copies.

Auguste Mariette avait une conscience certaine de l'influence de ce que l'on appellerait aujourd'hui la scénographie sur les visiteurs. L'exposition d'antiquités extra-européennes était alors une pratique ambiguë. Les antiquités classiques étaient surtout exposées en tant qu'œuvres d'art; il était donc normal de les présenter dans un cadre visuellement cohérent (néoclassique), et mieux encore d'exploiter leur environnement (perspective, espace, symétrie) pour magnifier leur effet et les mettre en valeur autant que possible d'un point de vue esthétique. En revanche, la situation était plus délicate lorsqu'il était question de vestiges

26. $1^{\text {re }}$ éd. Alexandrie, Mourès, Rey et $\mathrm{c}^{\mathrm{ie}}, 1864 ; 2^{\mathrm{e}}$ éd. Alexandrie, Mourès, Rey et $\mathrm{c}^{\mathrm{ie}}, 1868$; $3^{e}$ éd. Paris, A. Franck 1869 ; 4e éd. Alexandrie - Le Caire, Mourès et cie, 1872 (à partir de cette édition, « vice-roi » est remplacé par « khédive » dans le titre); $5^{e}$ éd. Le Caire, A. Mourès, $1874 ; 6^{e}$ éd. Le Caire, A. Mourès, 1876.

27. A. Mariette, Notice des monuments exposés dans les galeries provisoires du musée d'antiquités égyptiennes de S.A. le khédive à Boulaq, 6e éd., Le Caire, A. Mourès, 1876, p. 82.

28. Jean-François Champollion, Notice descriptive des monuments égyptiens du musée Charles $X$, Paris, Crapelet, 1827 : Emmanuel de Rougé, Notice sommaire des monuments égyptiens exposés dans les galeries du musée du Louvre, Paris, Vinchon, 1849 ; Paul Pierret, Musée du Louvre. Catalogue de la salle historique de la galerie égyptienne, Paris, Charles de Mourgues frères, 1873. 29. Samuel Sharpe, Egyptian Antiquities in the British Museum, Londres, John Russel Smith, 1862 ; S. Birch, op. cit. note 25.

30. Heinrich Brugsch, Uebersichtliche Erklærung ægyptischer Denkmæler des kœnigl. neuen Museums zu Berlin, Berlin, Ferd. Dümler, 1850 ; Karl Richard Lepsius, Koenigliche Museen. Abtheilung der Aegyptischen Alterthümer, Berlin, 1855. Dans cette dernière référence, rééditée en 1855 , il y a parfois une indication de provenance ou une attribution dynastique, mais de manière assez exceptionnelle.

31. Francesco Rossi, I monumenti egizi del museo d'antichità di Torino, Turin, Unione tipografico, 1884.

32. Les guides du musée de Boulaq ne sont pas pour autant exempts de défauts; à cause des réaménagements incessants, il était impossible de retrouver nombre d'objets évoqués. Un article remarqua avec pragmatisme : "[...] if the catalogue is full of omissions, erroneously indicates the place of the objects, entitles those objects according to their material and not their subject, and is without table of content and index [...], the visitor may console himself with the remembrance that there are Egyptian museums in Europe without catalog of any kind" ; « [...] si le catalogue est plein d'omissions, indique de façon erronée l'emplacement des objets, intitule ces objets selon leur matériaux et non leur sujet, et ne comporte pas de table des matières ni d'index [...], le visiteur peut se consoler en se rappelant qu'il y a en Europe des musées égyptiens sans catalogue d'aucune sorte », traduction de I'auteur ; Henry Wallis, « The Boulaq Museum », The Art-Journal, avril 1888 , pp. $103-110$, p. 110.

33. A. Mariette, Album du musée de Boulaq, Le Caire, Mourès et cie, 1872 ; Alexandre Brignoli, Musée égyptien. Album photographié, Le Caire, s. d. [1874 ?].

34. A. Mariette, Une visite au musée de Boulaq, Paris, A. Franck, 1869.

35. Eliott H. Colla, Conflicted Antiquities. Egyptology, Egyptomania, Egyptian Modernity, Durhan, Duke University Press, 2007, pp. 128-129. Hormis l'introduction du traducteur, le texte arabe traduit de Mariette n'a, à notre connaissance, pas été commenté, tandis que le texte français original n'est pas connu.

36. A. Mariette, Exposition de 1867. Description du parc égyptien, Paris, Dentu, 1867.

37. A. Mariette, La galerie de I'Égypte ancienne à l'exposition rétrospective du Trocadéro, Paris, Pichon, 1878

38. Les manipulations et des moulages non autorisés endommagèrent par exemple la statue de Kaâper, Musée égyptien du Caire CG 34, fig. 3 ; voir. H. Wallon, op. cit. note 19, pp. 143-144. 
archéologiques qui n'étaient pas issus du monde gréco-romain : il n'était pas facile de leur concéder une valeur artistique. En outre, l'égyptologie naissante était considérée comme une science historique ; on avait donc affaire à des documents qu'il fallait rendre lisibles et intelligibles (inscription, iconographie) plutôt qu’à des modèles esthétiques à magnifier. L'aménagement d'un musée égyptologique était donc une véritable question ${ }^{39}$. Quant à la décoration des salles, elle nallait pas de soi non plus. Les grands musées européens hésitaient entre le modèle néoclassique ${ }^{40}$ ou le style égyptianisant ${ }^{41}$. Auguste Mariette, ancien conservateur du Louvre, qui connaissait les musées de Londres, Berlin et Turin, a conçu le décor de son musée lui-même en composant avec les maigres moyens qui lui étaient confiés. Il copia les relevés archéologiques de Karl Richard Lepsius sur les murs des premières salles d'exposition ${ }^{42}$; par la suite il adopta pour le musée un décor discret mais coloré, composé notamment de frises à motifs égyptiens (lotus, " égyptiennes », etc.). Le mobilier était imité d'un meuble exhumé par les fouilles du Service ${ }^{43}$ et avait été réalisé pour l'Exposition universelle de Londres en 1862. Ces éléments participaient d'une véritable stratégie pour charmer les visiteurs.

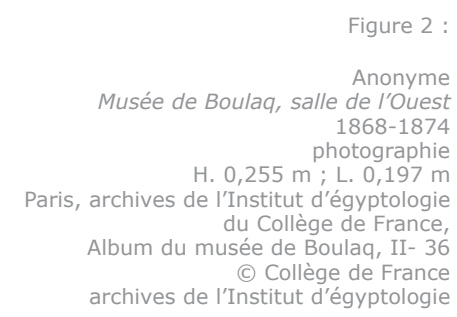

Figure 2 :
En effet, Auguste Mariette avouait dans l'avant-propos de sa Notice avoir recours à certains artifices pour séduire l'œil grâce à une disposition harmonieuse des objets ou la mise en valeur des chefs-d'œuvre du musée (fig. 2) : " [...] j’ai sacrifié au goût et cherché une certaine mise en scène qu'exclut ordinairement la froide régularité de nos Musées d'Europe ». Il s'en explique aussitôt : « [...] il est certain que, comme archéologue, je serais assez disposé à blâmer ces inutiles étalages qui ne profitent en rien à la science ; mais si le Musée ainsi arrangé plaît à

39. Pour cette problématique dans le cadre de la présentation des sculptures égyptiennes au British Museum au XIX ${ }^{e}$ siècle, voir Stephanie Moser, Wondrous Curiosities. Ancient Egypt at the British Museum, Chicago, University of Chicago Press, 2006.

40. Par exemple les salles de sculpture du British Museum (voir Ibidem, passim) ou le musée Charles X du Louvre (malgré les bas-reliefs égyptisants en trompe-I'œil et l'exotisme du décor, la structure reste classique).

41. Par exemple: les salles égyptiennes du Crystal Palace, voir Philip Henry Delamote, Photographic Views of the Progress of the Crystal Palace, Sydenham, Crystal Palace Company, [s. I.] 1855, pl. 68 ou le Neues Museum de Berlin en 1850.

Voir http://www.egyptian-museum-berlin.com/c01.php, Verein zur Förderung des Ägyptischen Museums Berlin e.V.

42. Édouard Mariette, Mariette Pacha. Lettres et souvenirs personnels, Paris, Jouve, 1904, pp. 180-181.

43. A. Rhoné, op. cit. note 6 , p. 80 . 
ceux auxquels il est destiné, s'ils y reviennent souvent et en y revenant s'inoculent, sans le savoir, le goût de l'étude et, j’allais presque dire, l'amour des antiquités de l'Égypte, mon but sera atteint. " ${ }^{44}$

\section{Le visiteur rêvé}

Toute cette stratégie pédagogique fonctionna admirablement sur Émile Guimet. Les remarques de son journal sont intéressantes. Dans le récit de sa première visite (le 28 novembre 1864), avant de détailler quelques éléments de la religion égyptienne, sa préoccupation principale, il remarque: "Le Musée est fort curieux, très-bien tenu et le catalogue, rédigé par M. Mariette, le conservateur, est admirablement fait ${ }^{45}$. " L'importance du "catalogue " dans l'appréciation de la visite est remarquable ; il précise avoir accompli sa visite "le catalogue à la main ${ }^{46}$ ". Il insère alors dans le récit de son voyage ce qu'il a appris sur l'Egypte ancienne. Il n'est pas anodin de noter qu'il reprend alors précisément les informations données par la Notice $e^{47}$. Ces similitudes prouvent que le guide s'est montré efficace dans son rôle; il est aussi probable qu'il ait servi a posteriori à combler les lacunes de la mémoire lors de la rédaction ${ }^{48}$.

Dans le récit de sa seconde visite, une dizaine de jours plus tard (le 9 décembre 1864), il formule une remarque également intéressante :

"Visite au musée de Boulak. Mariette-Bey, en l'organisant, a eu une très-bonne idée, qui consiste à indiquer pour chaque objet antique sa provenance, les circonstances dans lesquelles il a été retrouvé et, quand on le peut, l'époque à laquelle il remonte. De cette manière, chaque fragment a son intérêt et son enseignement. Tant que les musées d'antiquité ne suivront pas cette méthode, ils n'apprendront jamais rien aux visiteurs, tandis que le musée égyptien de Boulak est intéressant et attachant dans toutes ses parties ${ }^{49}$."

La stratégie d'Auguste Mariette avait fonctionné, au point même d'éveiller une vocation, comme l'avoua Émile Guimet lui-même quarante ans plus tard :

«En 1865, j'entreprenais, comme tout le monde, un voyage en Égypte. La vue des monuments, la visite au Musée de Boulacq, la lecture du merveilleux catalogue rédigé par Mariette, attrayant même pour les profanes, attachant comme un roman, les petits objets antiques qu'on se croit obligé de rapporter, tout cela avait ouvert mon esprit aux choses des temps passés et particulièrement aux croyances encombrantes dont les symboles se déroulent en Égypte sur des kilomètres de muraille ${ }^{50}$."

Après son voyage, Émile Guimet commença à collectionner les antiquités égyptiennes, spécialement ce qui touchait à la religion, tout en se documentant sur le sujet. En 1874, il possédait plus de quatre cent cinquante objets égyptiens dans sa collection privée ${ }^{51}$. Dans une optique comparatiste, il s'intéressa aussi à la Perse, l'Inde et l'Extrême-Orient. Il partit en 1876 au Japon, en Chine et en Inde

44. A. Mariette, Notice des monuments exposés dans les galeries provisoires du musée d'antiquités égyptiennes de S.A. le vice-roi à Boulaq, Alexandrie, Mourès, Rey et cie, 1864, pp. 7-8.

45. É. Guimet, op. cit. note 12, p. 55. Auguste Mariette n'était toutefois pas le conservateur mais le directeur du musée ; le conservateur titulaire était alors Luigi Vassalli.

46. Idem, Ibidem, p. 56.

47. Comparer les commentaires de la statue de Chéphren (Musée égyptien du Caire CG 14, fig. 1 au fond) : Ibid., pp. 281-282 et Auguste Mariette, op. cit. note 43, pp. 179-180 ; Émile Guimet reprend de la Notice le rappel que cette statue permet d'établir que les rois de la IV dynastie utilisaient l'écriture, ce qui n'était pas attesté peu de temps auparavant.

48. Émile Guimet n'avait pas noté dans son carnet les descriptions qui figurent dans le récit publié, Véronique Gay, « Croquis égyptiens. Émile Guimet en Égypte », Geneviève Galliano (dir.), Un jour j'achetai une momie. Émile Guimet et I'Égypte antique, Lyon, Hazan, 2012, pp. 34-35.

49. É. Guimet, op. cit. note 7, p. 124.

50. É. Guimet, Le jubilé du musée Guimet. Vingt-cinquième anniversaire de sa fondation. 18791904, Paris, Ernest Leroux, 1904, p. 10.

51. G. Galliano, « Histoire des collections égyptiennes d'Émile Guimet », op. cit. note 48, p. 46. 
pour poursuivre ses recherches et compléter sa collection, dans le cadre d'une mission sollicitée auprès du ministère de l'Instruction publique. Grâce à cette collecte, il créa en 1879 un Musée de l'histoire des religions et des civilisations orientales à Lyon, déplacé en 1889 à Paris (fig. 3) et devenu depuis, après le transfert de ses collections antiques au Louvre en 1948, le Musée national des arts asiatiques - Guimet.

Figure 3 :

Anonyme Musée de Boulaq, la statue de Kaâper dans la salle du Centre 1868-1883 H. 0,241 m: L. 0,177 m H. $0,241 \mathrm{~m} ; \mathrm{L}$. 0,177 m 'Institut d'égyptologie
du Collège de France sée de Boulaq, III-50 (c) Collège de France 
En 1889, presque trente ans après son ouverture, le musée de Boulaq fut fermé afin de transférer ses collections dans un lieu bien plus adapté, le palais de Giza ${ }^{52}$. En 1902, les collections, et la tombe d'Auguste Mariette - qui les avait suivies à Giza -, connurent un autre transfert vers l'actuel Musée égyptien du Caire. À l'instar du venin du canard égyptien, le travail d'Auguste Mariette pour transmettre son intérêt à l'antiquité égyptienne se révéla d'une redoutable efficacité. Les écrits d'Émile Guimet reconnurent son rôle déterminant dans sa propre vocation à fonder un musée; mais l'influence de Boulaq sur le musée Guimet ne s'arrêtait pas là. Certains dispositifs scénographiques du musée d'Auguste Mariette y furent en effet repris à l'identique, comme les meubles octogonaux à vitrine, ou même une des pièces emblématiques des collections de Boulaq, la statue de Kaâper, dont il exposa une copie ${ }^{53}$ dans sa galerie égyptienne (fig. 3 et 4). Enfin, les murs des deux salles égyptiennes étaient décorés des panneaux réalisés à la demande d'Auguste Mariette pour le pavillon égyptien de l'Exposition universelle de $1878^{54}$.

\section{L'auteur}

Après avoir obtenu en 2012 une licence d'histoire de l'art à l'université Paris 1 Panthéon - Sorbonne et le diplôme de $1^{\text {er }}$ cycle de l'École du Louvre, Thomas Lebée a réalisé son mémoire d'étude sur le musée de Boulaq. Il a ensuite travaillé, dans le cadre du M2 de l'École du Louvre, sur la collection des stèles égyptiennes tardives du Louvre, et à l'École pratique des Hautes Études sur l'évolution du dieu Seth au Ir millénaire av. J.-C.

52. G. Maspero, « Le musée de Boulaq et le musée de Guizéh », La nature 18-2, 1890, pp. 199202

53. Cette copie fut transférée en 1948 , avec toutes les collections égyptologiques du musée Guimet, au département des antiquités égyptiennes du musée du Louvre, où elle est toujours conservée sous le numéro d'inventaire E 20362. Nous remercions Élisabeth David de nous l'avoir signalé.

54. G. Galliano, op. cit. note 51, p. 46. 